\title{
Dip Constrained Migration Velocity Analysis and Interpretative processing
}

\author{
Luiz Alberto Santos (PETROBRAS/UFF), Eduardo Filpo Ferreira da Silva (PETROBRAS), Jessé C. da Costa (UFPA)
}

Copyright 2019, SBGf - Sociedade Brasileira de Geofísica

This paper was prepared for presentation during the $16^{\text {th }}$ International Congress of the Brazilian Geophysical Society held in Rio de Janeiro, Brazil, 19-22 August 2019.

Contents of this paper were reviewed by the Technical Committee of the $16^{\text {th }}$ International Congress of the Brazilian Geophysical Society and do not necessarily represent any position of the SBGf, its officers or members. Electronic reproduction or storage of any part of this paper for commercial purposes without the written consent of the Brazilian Geophysical Society is prohibited.

\section{Abstract}

In pre-stack depth migration processing flow, there is the important step of velocity estimation. Migration velocity Analy sis (MVA) is one of most used method in the seismic industry. In this work we describe the MVA workflow that takes into account the dip of layers to automatically increase geologic information as a regularization procedure in inversion. We apply the workflow in a real data set from Campos Basin detailing the shallow region. The experiment clearly shows the importance of interpretation team interacting during processing. Particularly for the studied area, MVA deliv ers velocity field consistent with geologic ev olution of the site.

\section{Introduction}

Seismic workflow in oil industry is composed of three main phases: acquisition, processing and interpretation. Usually those phases have sharp limits. In pre-stack depth migration processing flow, there is the important step of velocity estimation. Migration velocity Analysis (MVA) is one of the most used method in seismic industry. The objective of MVA workflow (Figure 1) is the estimation of a velocity field for depth migration for best imaging.

Sometimes best imaging does not mean geologically f easible v elocity model. This subject has been addres sed by some authors. Delprat-Janaud and Lailly (1992) compute numerical uncertainties that approximate the phy sical uncertainties. They limit the study to Hilbertian model spaces and derive necessary and sufficient condition which yields the desired result - the norm chosen in model space has to bind the Frechet deriv ativ e of the forward map. Clapp et al. (2002) use nonstationary operators that tend to spread information along structural dips of layers in tomographic process. Costa et al. (2008) propose a reflection-angle-based kind of smoothness constraint as regularization in slope tomography. Santos et al. (2013) quantify the gradients dif ferences of velocity and amplitude volumes in a parameter called Geological Incoherence Index (GII). Luo et al. (2017) propose anisotropic diffusion smoothing operators into the conjugate gradient algorithm to precondition tomography.
We developed and apply the structure tensor based regularization in MVA process using a workflow that includes available geological information. With an example of Campos Basin dataset we show advantages and limitation of this process.

\section{Method}

The objective of the Migration Velocity Analysis (MVA) workf low (Figure 1) is the estimation of a v elocity field $f$ or depth migration for best imaging. In seismic reflection a point in underground may be sampled by different shot gathers. After migration, if we use the correct velocity field, the image point may be located at the same position $(x, y, z)$. The difference of event positions may be measured comparing common of set gathers (COG) (Trier (1990) and Deregowski (1990)) or evaluating common reflection points (CRP) as published in Al-Yahy a (1989), Symes e Carazzone (1991) and Jin and Madariaga (1994). Events at CRP gathers become flat when we use correct velocity field or a kinematically equiv alent v elocity field (Santos et al, 2013).

A starting velocity field is used to migrate the seismic data. After migration, step 2, the data is organized in CRP-gathers. The flatness of the events are used as a metric for Stopping Criteria step. If the events are flat, the velocity field is accepted $(\mathrm{Y})$ and the process stops delivering the suitable velocity field for imaging. Otherwise, if the events are not flat $(\mathrm{N})$ the process continues.

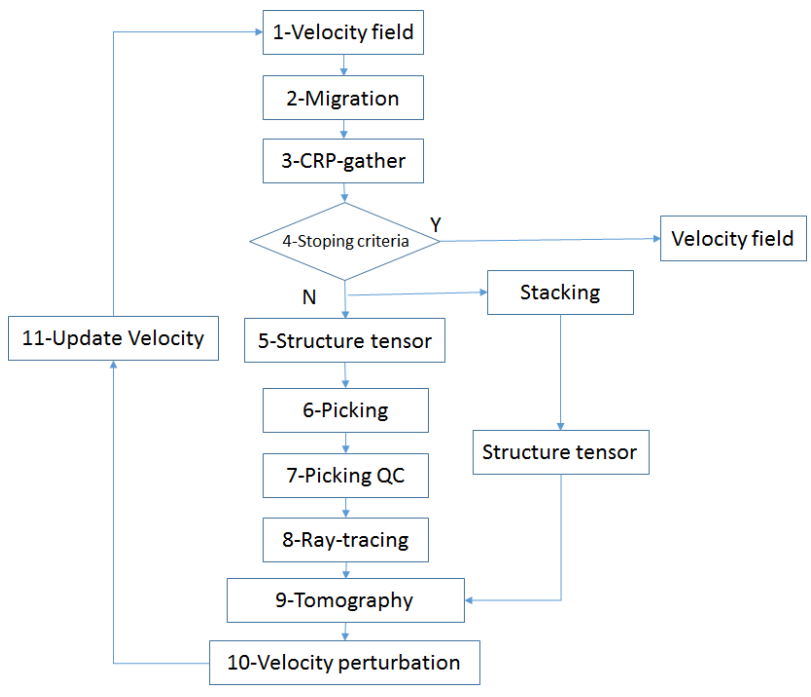

Figure 1: MVA workf low 
In the main stream of the MVA process, in step 5, structure tensor (Fehmers and Christian, 2003) of CRPgather are calculated. Picking makes use of CRPamplitude gathers and CRP-gathers structure tensor (Silv a, 2019). In step 7, picking QC, av oids non feasible pickings.

In step 8, rays are traced in the current velocity field. Ray trajectories are used to build the sensitivity matrix. In step 9 , Tomography, sensitiv ity matrix and the misf it of picked events are used to calculate velocity perturbation employing a Gauss-Newton based algorithm. This perturbation is algebraically added to current v elocity field in step 11. Then, back to step 1, the workf low runs again until the stopping criteria is reached.

The main branch of the workflow does not assure an estimation of a feasible velocity field, even in a convergent inversion process. In practice, the main branch makes use of a simple objective function $\Phi(m)$ (equation 1).

$$
\Phi(m)=\|d-F(m)\|_{2}^{2}
$$

In MVA $d$ is the depth of the event at of fset zero. The objective function measures the misfit between the calculated depth in the migration process at each of $f$ set, $F(m)$, and its depth at of $f$ set zero.

The iterative solution, so far, may converge to an unf easible velocity field. This subject has already been discussed and treated by some authors (Delprat-Janaud and Lailly (1992), Clapp et al. (2002), Costa et al. (2008), Santos et al. (2013) and Luo et al. (2017)).

In this work, a parallel branch of MVA workflow is performed to regularize and guide the convergence to a solution more feasible geologically. This secondary branch (Figure 1) starts just after the Stopping Criteria step. CRP-gathers are stacked delivering a seismic volume. Then, Structure tensor of this volume is calculated and the results are used to regularize the inversion in the Tomography step.

The structure tensor is perf ormed to calculate an operat or $D_{R}$ to use for regularization in objectiv e function:

$$
\Phi(m)=\|d-F(m)\|_{2}^{2}+\lambda_{r}\left\|D_{R}\left(m-m_{r}\right)\right\|_{2}^{2} .
$$

where $\lambda_{r}$ weight the regularization and $m_{r}$ is a ref erence model vector.

This parallel flow is paramount to estimate velocity field that follows layering and secondary structures of underground.

All the routines used in the described workflow, Figure 1, are proprietary programs dev eloped in Petrobras.

\section{Application}

We apply the described workflow, Figure 1, in a dataset surveyed in a sector of Campos Basin. The data were previously processed using post-stack time migration flow. As there are sea bottom cany ons in the surveyed area, the available starting velocity for MVA is not suitable. It was deriv ed from a NMO velocity cube, whose the premises are corrupted by the lateral velocity contrast at canyons. Then, we needed to build another velocity field.

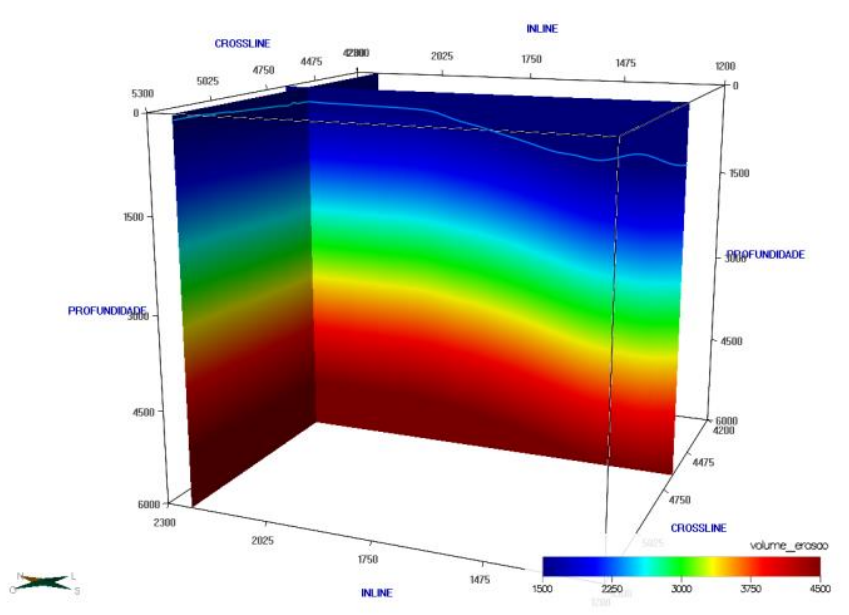

Figure 2: Starting v elocity field built in V3O2 sy stem.

According to Rosa (2010), Campos Basin vertical velocity gradient for clastic section is $0,6 \mathrm{~s}^{-1}$. For the target area, the starting velocity just below sea bottom is unknown. For such shallow region, it depends on the sedimentation and erosive episodes. In the absence of feasible inf ormation, we used $1500 \mathrm{~m} / \mathrm{s}$ as the sediment velocity at the sea bottom.

The starting velocity model is created with $1500 \mathrm{~m} / \mathrm{s}$ for the water column and a sea bottom concordant gradient of $0.6 \mathrm{~s}^{-1}$ - Figure 2 . The model is created in $\mathrm{V} 3 \mathrm{O} 2-\mathrm{a}$ Petrobras proprietary system for seismic interpretation, data integration and seismic inversion. In this procedure, we used a smooth version of sea bottom to av oid sharp marks in the initial velocity field and to simulate the ef $f e c t$ of erosion in the initial velocity field. Erosion exposes deeper sediments with higher velocities. Using a smooth version of sea bottom to calculate velocity gradient and updating the model above actual sea bottom with water velocity $(1500 \mathrm{~m} / \mathrm{s})$ simulates erosion effect. After this procedure, we create the first v elocity model $-m_{0}$ - Figure 2.

Depth migration is performed with narrow aperture, $2 \mathrm{Km}$, to resolve shallow depths. After migration with $\mathrm{m}_{0}$ model, we obtain the result of Figure 3.

The resulted migrated volume and CRP (Figure 3) point to under-estimation of the velocity field $\mathrm{m}_{0}$. So, in Step 4 current velocity field was not accepted and inversion process continued.

Structure tensor (step 5) is calculated over each CRPgather and, then, picking process starts (step 6). Picking 
tracks events on CRPs using the method described in Silva (2019). After picking, during QC step, complex curves are avoided and the remaining ones are stored. Over the current velocity field, rays are traced to build sensitiv ity matrix employ ed in Tomography, step 9. In this step, the misfit between each stored curve and the expected behavior, flat ev ent in CRP, is used to com pute the first term of equation 2. Also, parallel calculation of structure tensor is done over the migrated volume to be used in regularization - second term right hand side of equation 2. In this application we employ the current model as the ref erence one $\left(\mathbf{m}_{\mathbf{r}}\right)$.

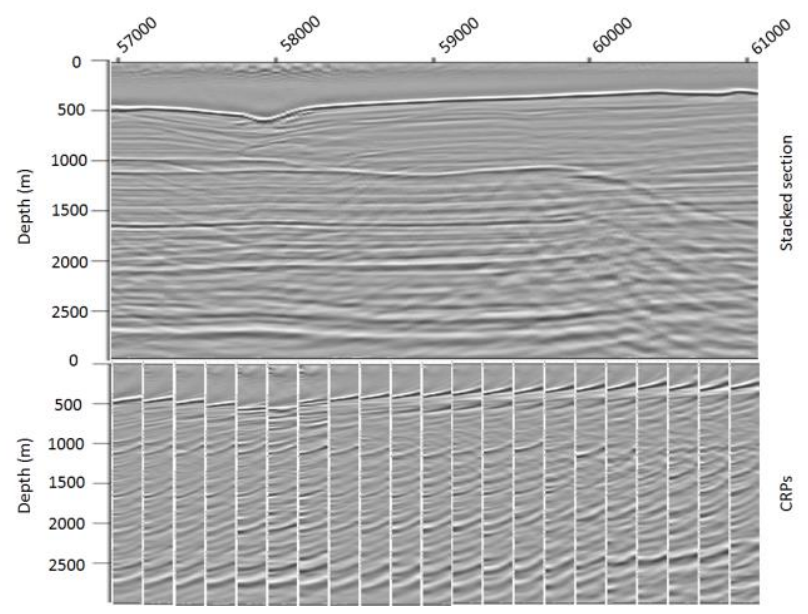

Figure 3:Top: Migrated section with $\mathrm{m}_{0}$ and; Bottom: Corresponding CRPs migrated with the starting velocity field (Figure 2).

The perturbation in velocity field in this first iteration shows structures of underground, due to the employed regularization - equation 2. Lay ering of thicker strata is imposed in the perturbed velocity model. The calculated incremental velocity shows mainly positiv e values close to sea bottom that av erage 15 to $30 \mathrm{~m} / \mathrm{s}$. (Figure 4). Finally, it is remarkable that higher positive values are below and along the borders/shoulders of cany ons ranging from 35 to $50 \mathrm{~m} / \mathrm{s}$.

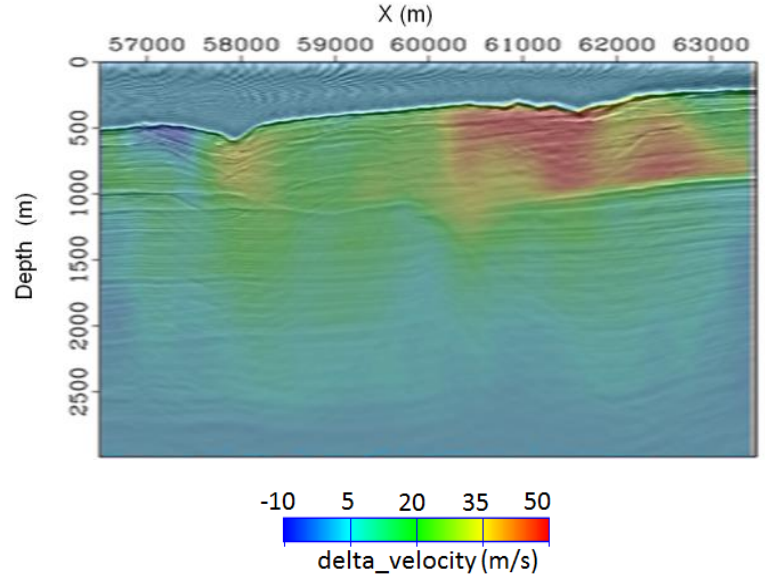

Figure 4: Overlay of the perturbation of velocity after the first iteration.

After the second iteration the pattern of the velocity structure perturbation resembles the one in the first iteration (Figure 4) in the shallow part, shallower than $1000 \mathrm{~m}$. This pattern of progressive increase of velocity field at each iteration means we underestimate sediment velocity at sea bottom.

\section{Results}

The MVA process converged to a model showing more complexities in the shallow part of the volume (Figure 5). Those complexities, once the velocity is well estimated, promotes better imaging at deeper depths. Bey ond imaging, the inversion process, as a phase in the seism ic processing macro-workflow, brings important inputs for interpretation.

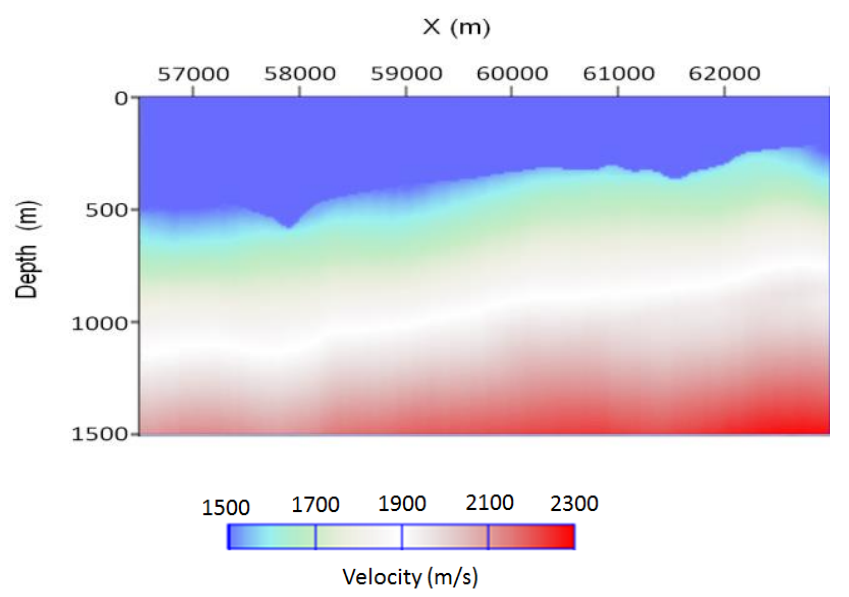

Figure 5: Final velocity model for the shallow region (shallower than $1000 \mathrm{~m}$ ).

The inversion system show that the initial velocity is underestimated. Along the entire inverted volume, v elocity at sea bottom is higher than $1520 \mathrm{~m} / \mathrm{s}$. Sea bottom velocities inside canyons are even higher, which increases the velocity gradient from the water layer to sediment pile and locally reaching $1600 \mathrm{~m} / \mathrm{s}$. This characteristic brings information about cany on ev olution. Considering velocities proportional to compaction of sediment pile and age, in this region, there was no recent filling episode of both cany ons. They are just excav ating the sea bottom and exposing old and more compacted sediments.

Geometry of shallow events in migrated seismic data agrees with the information provided by MVA process. The regularization using structure tensor - second term of right hand side of equation 2 - calculate geologically feasible velocity field.

\section{Discussion}

The described process shows the inclusion of geologic information in MVA. Geologic information about 
compaction gradient is successfully used giving an initial v elocity able to converge to reasonable solution.

The absence of information - the sediment velocity at sea bottom - is corrected during the tomographic process and it shows the robustness of the inversion system. The inversion process also brings geological valuable inf ormation by the velocity structure, indeed enhanced by using structure tensor guided regularization.

We conf ine our analy sis along the shallow section of the data volume. We reach such good results because we do not ask to the data, more than it really can bring. As the migration aperture is $2 \mathrm{Km}$, we restricted conf idence in the results up to $1000 \mathrm{~m}$. CRPs below that depth are not flat.

Despite estimated velocity field is brought in low spatial frequency, it is the first elastic property to reveal underground characteristics for exploration and/or production purposes. The MVA process needs and delivers geologic information that claims interpreters interaction. Quantitativ e interpretation during processing.

\section{Conclusions}

Convergence is reached using geological knowledge of underground. It is better than the velocity field from PSTM processing flow because of cany ons damaging velocity semblance analy sis in time domain.

Structure tensor is a very important step to estimate geologically feasible velocity field.

Interpretation needs to start during MVA as it brings and receives $v$ aluable information about subsurface reliable compressional velocities.

Particularly for this area, the canyons show no recent episode of sediment filling. They are still in an erosive phase. Geological knowledge and inversion process reach coherently the same conclusion.

\section{Acknowledgments}

We would like to thanks Petrobras that allows the publication of this work and all colleagues of the Exploration Team.

\section{References}

Al-Yahy a, K., 1989, Velocity analy sis by iterative profile migration: Geophy sics, 54, 718-729.

Clapp, R.G., Biondi, B., Claerbout, J., 2004, Incorporating geologic information into reflection tomography: Geophy sics, 69, 533-546.

Costa, J. C., Silva, F.J.C., Gomes, E.N.S., Schleicher, J., Melo, L.A.V., Amazonas, D., 2008, Regularization in slope tomography: Geophy sics, 73, VE39-VE47.

Delprat-Jannaud, F. and LAILLY, P., 1992, What Information on the Earth Model Do Reflection Travel
Times Provide?: Journal of Geophy sical Research, 97, 19827-19844.Deregowski (1990))

Fehmers, G.C. and Christian, F.W.H., 2003, Fast structural interpretation with structure-oriented filtering: Geophy sics, 1286-1293.

Jin, S., and Madariaga, R., 1994, Nonlinear velocity inv ersion by a two-step monte carlo method: Geophy sics, 59, 577-590.

Luo, Z., Fan, D., Farmer, P., Martin, G., 2017, Obtaining geologically conformable tomographic models through anisotropic diffusion preconditioning: 87th Annual International Meeting, SEG, Expanded Abstracts, 56915695.

Rosa, A.L.R., 2010, Análise do Sinal Sísmico: Sociedade Brasileira de Geof ísica.

Santos, L.A., Mansur, W.J., McMechan, G.A., 2013, Geologic incoherence index for seismic inversion: 13rd International Congress of the Brazilian Geophysical Society, Expanded Abstracts.

Silva, E.F.F., 2019, On the application of the image guided ray-tracing: 16th International Congress of the Brazilian Geophy sical Society, Expanded Abstracts.

Symes, W.W., and CARAZZONE, J.J., 1991, Velocity inversion by differential semblance optimization: Geophy sics, 56, 654-663.

Trier, J. A., 1990, Tomographic determination of structural velocities from depth-migrated seismic data: PhD thesis, Stanf ord Univ ersity. 